\title{
IMPACT RESPONSE OF BRIDGES WITH ELASTIC BEARINGS TO MOVING LOADS
}

\author{
J.-D. YAU \\ Department of Architecture and Building Technology, Tamkang University, Taipei, Taiwan 106 \\ AND \\ Y.-S. Wu, Y.-B. YANG \\ Department of Civil Engineering, National Taiwan University, Taipei, Taiwan 10617. \\ E-mail:vbyang@ce.ntu.edu.tw
}

(Received 22 August 2000, and in final form 17 January 2001)

\begin{abstract}
In this paper, the dynamic response of bridge girders with elastic bearings to moving train loads is studied using an analytical approach. Such a problem hàs not been very well investigated in the literature. The present results indicate that the dynamic response of the beam at resonance remains generally constant, if the effect of damping is taken into account, and that the insertion of elastic bearings at the supports of the beam for the purpose of isolating the earthquake forces may adversely amplify the dynamic response of the beam to moving train loads. An envelope impact formula is derived for the deflection of the beam with light damping, which serves as a useful, preliminary design aid to railway engineers.

(C) 2001 Academic Press
\end{abstract}

\section{INTRODUCTION}

To prevent the damage of bridges from severe earthquakes, elastic bearings are often adopted as base isolators in bridge engineering. Conventionally, they are installed at the supports of bridge girders to isolate the vibration energy transmitted from the ground. However, such devices may adversely result in amplification of the response of the bridge girder to moving train loads. In this study, an analytical approach is presented for investigating the dynamic response of bridges with elastic bearings to a series of moving loads to simulate the action of high-speed trains. Of particular interest is the resonant response that may be induced on the bridge.

The dynamic response of bridge structures to moving loads at high speeds is a problem of great concern in the design of high-speed railway bridges. In the literature, a larger number of analytical investigations have been carried out, with the bridge modelled as a beam-like structure and the vehicles as moving loads or moving masses [1-4]. Recently, Yang et al. [5] presented a closed-form solution for the dynamic response of simple beams subjected to a series of moving loads at high speeds, in which the phenomena of resonance and cancellation have been identified, along with optimal design criteria proposed. By considering the effect of damping, $\mathrm{Li}$ and $\mathrm{Su}$ [6] investigated the fundamental characteristics and dominant factors for the resonant vibration of a girder bridge under high-speed trains. Using the dynamic stiffness approach and damped Timoshenko beam theory, Chen and Li [7] investigated the dynamic response of some elevated high-speed railways considered by the High-Speed Rails Bureau of Taiwan for the preliminary design. 
On the other hand, based on the finite element methods, more sophisticated models have been devised to study the dynamic behavior of vehicle-bridge interaction problems by researchers. To resolve the coupling effect between the bridge and moving vehicles, methods that are of iterative nature have been employed [8-10]. By the concept of dynamic condensation, Yang and co-workers $[11,12]$ developed the vehicle-bridge interaction (VBI) elements for the dynamic analysis of railway bridges subjected to moving trains. Cheung et al. [13] used the modified beam vibration functions to investigate the response of multi-span non-uniform bridges under moving vehicles and trains. Ichikawa et al. [14] used the modal analysis method to study the dynamic behavior of the continous beam subjected to a moving mass. They found that the inertial effect of moving mass has a greater influence on the second and successive spans than on the first span.

To the knowledge of the authors, very few studies have been conducted on the dynamic response of elastically supported beams to moving loads. The objective of this paper is to analytically formulate the dynamic problem of elastically supported beams subjected to high-speed moving loads. One advantage with the analytical approach is that the key parameters governing a physical response can be identified. However, it forbids us to use physical models that are too complicated. For this reason, no attempt will be made herein to consider effects such as vehicle-bridge interaction and vehicle damping. Based on the present results, an envelope impact formula will be proposed for the deflection of the beam with the structural damping taken into account. The accuracy of the formula will be demonstrated in the numerical examples through comparison with the finite element solutions.

\section{EQUATION OF MOTION}

As shown in Figure 1, a beam supported by two elastic bearings of stiffness $K$ at the two ends is considered. The beam is assumed to have a length $L$ and uniform cross-sections. The train moving over the beam at speed $v$ is modelled as a sequence of equidistant moving loads. The interval between two adjacent moving loads is $d$ and the weight of each moving load is $p$. The equation of motion for the beam travelled by the moving loads can be written as [5]

$$
m \ddot{u}+c_{e} \dot{u}+c_{t} \dot{u}^{\prime \prime \prime \prime}+E I u^{\prime \prime \prime \prime}=p \sum_{k=1}^{N} \delta\left[x-v\left(t-t_{k}\right)\right] \times\left[H\left(t-t_{k}\right)-H\left(t-t_{k}-L / v\right)\right],
$$

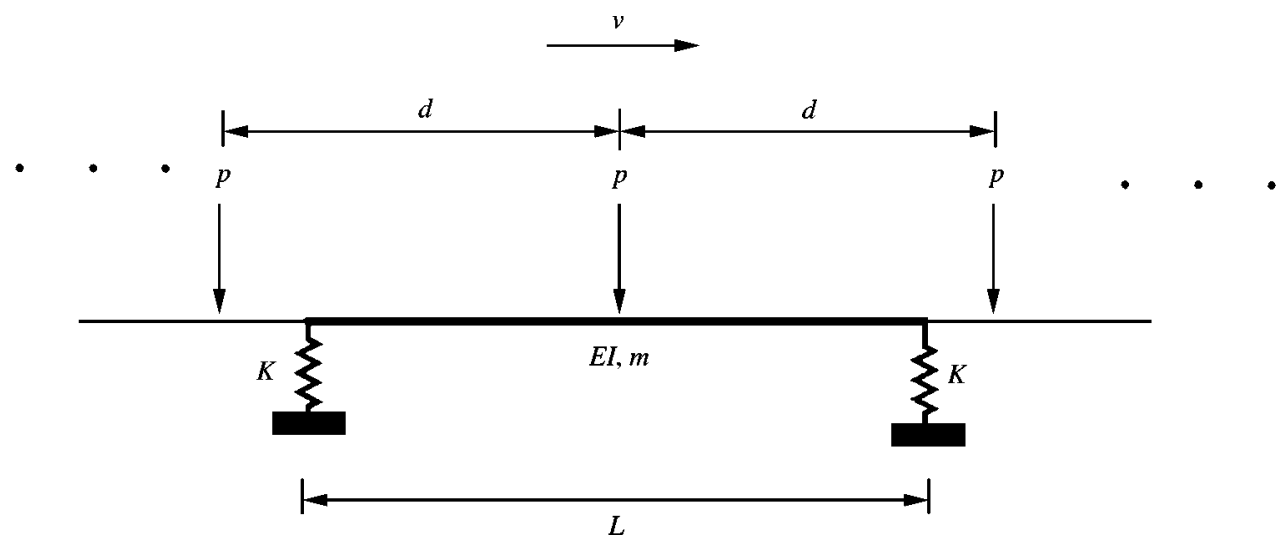

Figure 1. Elastically supported beam subjected to uniform moving loads. 
where a prime denotes derivative with respect to the co-ordinate $x$, an overdot denotes derivative with respect to time $t, m$ is the mass per unit length of the beam, $u(x, t)$ the vertical displacement, $c_{e}$ the external damping coefficient, $c_{i}$ the internal damping coefficient, $E$ the elastic modulus, $I$ the moment of inertia, $\delta$ the Dirac delta function, $H(t)$ the unit step function, $N$ the total number of moving loads, and $t_{k}=(k-1) d / v$ is the arriving time of the $k$ th load at the beam. Correspondingly, the boundary conditions of the beam are

$$
\begin{aligned}
& E I u^{\prime \prime}(0, t)=E I u^{\prime \prime}(L, t)=0, \\
& E I u^{\prime \prime \prime}(0, t)=-K u(0, t), \\
& E I u^{\prime \prime \prime}(L, t)=K u(L, t)
\end{aligned}
$$

and the initial conditions are

$$
u(x, 0)=\dot{u}(x, 0)=0
$$

assuming the beam is initially at rest.

\section{FUNDAMENTAL FREQUENCY OF THE BEAM}

To analyze the dynamic response of the elastically supported beam to a sequence of moving loads, the vibration shape of the beam will be approximated by the combination of a flexural sine mode and a rigid displacement mode, as shown in Figure 2. Thus, the

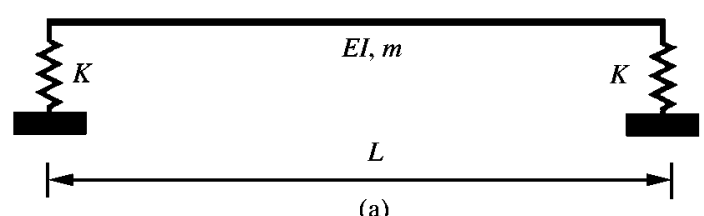

(a)
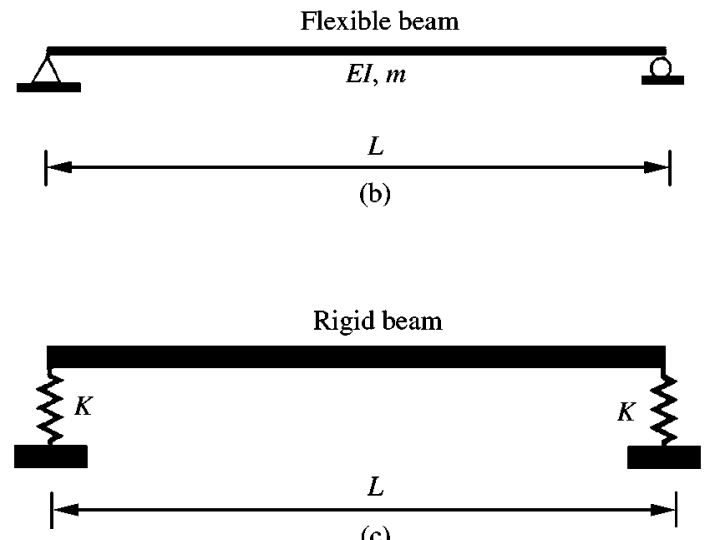

(c)

Figure 2. Model of free vibration shape of elastically supported beam. 


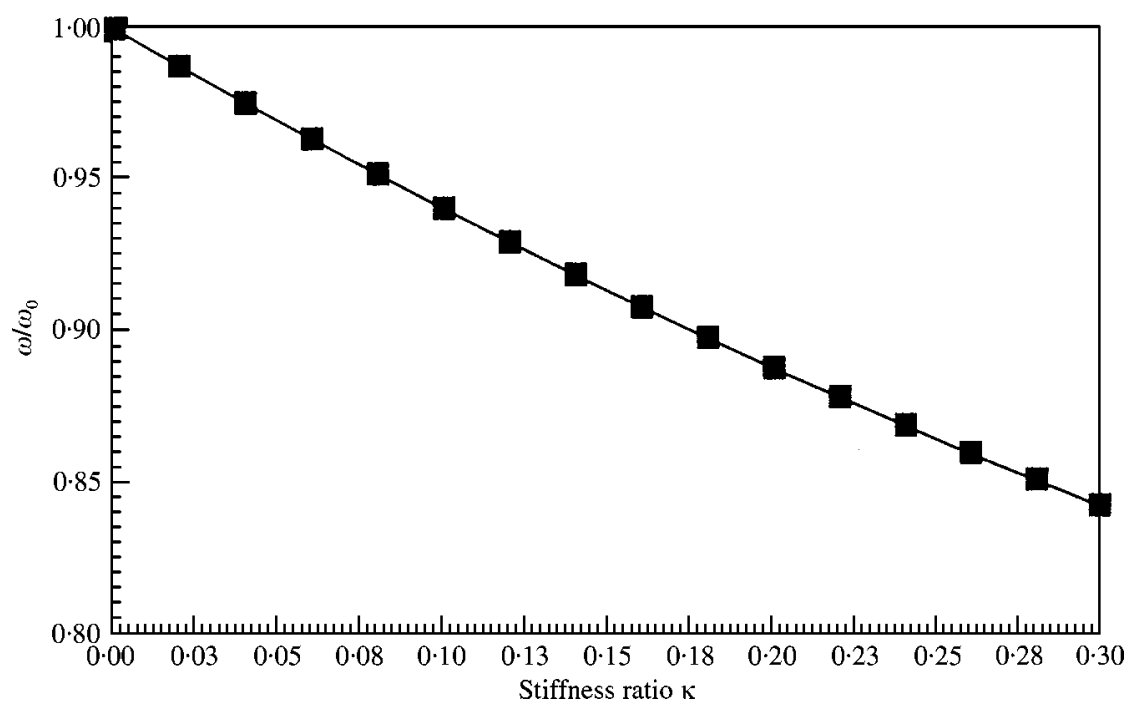

Figure 3. Comparison of fundamental frequencies. - Exact solution; $\mathbf{\square}$, Present.

displacement $u(x, t)$ of the elastically supported beam can be expressed as

$$
u(x, t)=q(t) \phi(x) \cong q(t) \times \frac{\sin (\pi x / L)+\kappa}{1+\kappa},
$$

where $q(t)$ denotes the generalized co-ordinate of the vibration shape, $\phi(x)$ the assumed shape function, and $\kappa\left(=E I \pi^{3} / K L^{3}\right)$ is the stiffness ratio of the beam to the elastic bearings. In particular, the term $\kappa$ in the numerator of equation (4) denotes the rigid displacement mode. For the special case with $\kappa=0$, which implies hinged supports, the assumed mode shape $\phi(x)$ reduces to the flexural mode. As can be seen, higher modes other than the first flexural and rigid modes have been excluded from equation (4), which is justified for the moving load problems, because of the transient nature of the beam in response to the moving loads, and is particularly true if only the midpoint displacement of the beam is desired.

By Rayleigh's method, the fundamental frequency $\omega$ of the elastically supported beam can be computed as

$$
\omega^{2}=\frac{\int_{0}^{L} E I\left[\phi^{\prime \prime}(x)\right]^{2} \mathrm{~d} x+K\left\{[\phi(0)]^{2}+[\phi(L)]^{2}\right\}}{\int_{0}^{L} m[\phi(x)]^{2} \mathrm{~d} x} \cong \frac{\omega_{0}^{2}(1+4 \kappa / \pi)}{1+8 \kappa / \pi+2 \kappa^{2}},
$$

where $\omega_{0}=(\pi / L)^{2} \sqrt{(E I / m)}$ is the fundamental frequency of the corresponding beam with hinged supports. When $\kappa$ equals 0 , the fundamental frequency $\omega$ of the elastically supported beam becomes equal to that of the corresponding simple beam. On the other hand, if $\kappa$ approaches infinity, the frequency $\omega$ reduces to zero, meaning that the beam is unsupported. To verify the accuracy of the approximate fundamental frequency given by equation (5), the exact solution will be computed from the following frequency equation for 
the elastically supported beam [15]:

$$
b^{2}\left(\cos k L-\frac{1}{\cosh k L}\right)+2 b(\sin k L-\tanh k L \cos k L)-2 \sin k L \tanh k L=0,
$$

where $k \mathrm{~L}=\pi \sqrt{\left(\omega / \omega_{0}\right)}$ and $b=E I k^{2}$. The fundamental frequencies computed from equation (5) have been compared with the exact ones solved from equation (6) for different stiffness ratios in Figure 3. As can be seen, the approximate frequencies agree excellently with the exact ones, implying that the use of the approximate mode shape for the elastically supported beam, as given in equation (4), is acceptable.

\section{DYNAMIC RESPONSE ANALYSIS}

By substituting the expression for the displacement $u(x, t)$ in equation (4) into equation (1), multiplying both sides of the equation by the shape function $\phi(x)$, and then integrating with respect to the beam axis $x$ over the length $L$, one obtains the equation of motion in terms of the generalized co-ordinate $q(t)$ as

$$
\ddot{q}(t)+2 \xi \omega \dot{q}(t)+\omega^{2} q(t)=\frac{2 p(1+\kappa)}{m L\left(1+8 \kappa / \pi+2 \kappa^{2}\right)} \sum_{k=1}^{N} F_{k}(v, t),
$$

where $\xi$ is the modal damping ratio and $F_{k}(v, t)$ is the generalized forcing function.

$$
F_{k}(v, t)=\left[\kappa+\sin \Omega\left(t-t_{k}\right)\right] H\left(t-t_{k}\right)+\left[-\kappa+\sin \Omega\left(t-t_{k}-\frac{L}{v}\right)\right] H\left(t-t_{k}-\frac{L}{v}\right) .
$$

Here, $\Omega(=\pi v / L)$ is the driving frequency, as implied by the moving loads.

First, consider the case when only a single moving load is crossing the bridge. The equation of motion (7) becomes

$$
\ddot{q}(t)+2 \xi \omega \dot{q}(t)+\omega^{2} q(t)=\frac{2 p(1+\kappa)}{m L\left(1+8 \kappa / \pi+2 \kappa^{2}\right)}\left(\sin \frac{\pi v t}{L}+\kappa\right) .
$$

By Duhamel's integral, the generalized co-ordinate $q(t)$ can be solved from equation (9) as

$$
\begin{aligned}
q(t)= & \frac{\Delta_{s t}(1+\kappa) /(1+4 \kappa / \pi)}{\left(1-S^{2}\right)^{2}+(2 \xi S)^{2}} \\
& \times\left\{\left(1-S^{2}\right) \sin \Omega t-2 \xi S \cos \Omega t+\mathrm{e}^{-\xi \omega t}\left[2 \xi S \cos \omega_{d} t-\frac{S\left(1-S^{2}-2 \xi^{2}\right)}{\sqrt{1-\xi^{2}}} \sin \omega_{d} t\right]\right\} \\
& +\frac{\Delta_{s t}(1+\kappa)}{1+4 \kappa / \pi} \times \kappa\left\{1-\mathrm{e}^{-\xi \omega t}\left[\cos \omega_{d} t+\xi \sqrt{1-\xi^{2}} \sin \omega_{d} t\right]\right\}
\end{aligned}
$$

where $\Delta_{s t}=2 p L^{3} /\left(\pi^{4} E I\right) \approx p L^{3} /(48 E I)$ is the maximum static deflection of the corresponding simple beam, $S=\Omega / \omega$ the speed parameter, and $\omega_{d}=\omega \sqrt{\left(1-\xi^{2}\right)}$ the damped frequency. It should be noted that the speed parameter $S$ represents the ratio of the driving frequency to the frequency of the beam. For most of the vehicle-bridge problems encountered in practice, the speed parameter $S$ is less than $0 \cdot 3$.

In this study, only elastically supported beams with light damping $(\xi<0.05)$ are considered, which implies that terms involving $\xi^{2}, \xi S$, and $\xi \kappa$ can be neglected. As a result, 
the response in equation (10) reduces to

$$
q(t) \cong \frac{\Delta_{s t}(1+\kappa)}{1+4 \kappa / \pi}\left[\frac{1}{1-S^{2}} G_{1}(v, t)+\kappa G_{2}(t)\right] \quad 0 \leqslant v t \leqslant L,
$$

where, noting that $\omega_{d} \cong \omega$, the functions $G_{1}(v, t)$ and $G_{2}(t)$ are given as

$$
\begin{aligned}
& G_{1}(v, t)=\sin \Omega t-S \mathrm{e}^{-\xi \omega t} \sin \omega t, \\
& G_{2}(t)=1-\mathrm{e}^{-\xi \omega t} \cos \omega t .
\end{aligned}
$$

Consider the case when a series of moving loads of constant intervals $d$ are crossing the bridge. The dynamic response can be extended from equation (11) as follows:

$$
\begin{aligned}
q(t) \cong & \frac{\Delta_{s t}(1+\kappa)}{1+4 \kappa / \pi}\left\{\frac{1}{1-S^{2}} \sum_{k=1}^{N}\left[G_{1}\left(v, t-t_{k}\right) H\left(t-t_{k}\right)+G_{1}\left(v, t-t_{k}-\frac{L}{v}\right) H\left(t-t_{k}-\frac{L}{v}\right)\right]\right. \\
& \left.+\kappa \sum_{k=1}^{N}\left[G_{2}\left(t-t_{k}\right) H\left(t-t_{k}\right)-G_{2}\left(t-t_{k}-L / v\right) H\left(t-t_{k}-L / v\right)\right]\right\},
\end{aligned}
$$

where $t_{k}=(k-1) d / v$ denotes the arriving time of the $k$ th load on the bridge, the unit step function $H\left(t-t_{k}\right)$ is used to represent the direct action of the $k$ th moving load on the beam, and the function $H\left(t-t_{k}-L / v\right)$ the residual action of the $k$ th moving load.

\section{PHENOMENA OF RESONANCE AND CANCELLATION}

In this study, the span length $L$ of the beam is assumed to be no greater than twice the interval $d$ between two consecutive moving loads, i.e., $L \leqslant 2 d$, which is the case implied by most high-speed railway constructions. In order to derive the conditions for the phenomena of resonance and cancellation to occur under the action of a series of moving loads, we shall neglect the effect of damping of the beam in this section. For this special case, $\xi=0$, the dynamic response of the beam in equation (13) reduces to

$$
q(t)=\frac{\Delta_{s t}(1+\kappa)}{1+4 \kappa / \pi}\left[\frac{P_{1}(v, t)}{1-S^{2}}+\kappa P_{2}(v, t)\right]
$$

where

$$
\begin{aligned}
P_{1}(v, t)= & \sum_{k=1}^{N}\left\{\left[\sin \Omega\left(t-t_{k}\right)-S \sin \omega\left(t-t_{k}\right)\right] H\left(t-t_{k}\right)\right. \\
& \left.+\left[\sin \Omega\left(t-t_{k}-L / v\right)-S \sin \omega\left(t-t_{k}-L / v\right)\right] H\left(t-t_{k}-L / v\right)\right], \\
P_{2}(v, t)= & \sum_{k=1}^{N}\left\{\left[1-\cos \omega\left(t-t_{k}\right)\right] H\left(t-t_{k}\right)-\left[1-\cos \omega\left(t-t_{k}-L / v\right)\right] H\left(t-t_{k}-L / v\right)\right\} .
\end{aligned}
$$

In equation (14) and (15), the function $P_{1}(v, t)$ indicates the contribution of the flexural vibration mode of the simple beam, and $P_{2}(v, t)$ the rigid displacement mode of the elastic bearings. The beam will be excited to its maximum, in the sense that the response will reach 
the maximum, when the last (i.e., the $N$ th) moving load in the sequence enters the beam. For this case, $t_{N}<t<t_{N}+L / v$, the function $P_{1}(v, t)$ in equation (15a) can be simplified as [5]

$$
\begin{aligned}
P_{1}(v, t)= & {\left[\sin \Omega\left(t-t_{N}\right)-S \sin \omega\left(t-t_{N}\right)\right] H\left(t-t_{N}\right)-2 S \cos \frac{\omega L}{2 v} \times\left[\sin \omega\left(t-\frac{L}{2 v}\right)\right.} \\
& \left.+\sin \omega\left(t-\frac{t_{N}+L / v}{2}\right) \frac{\sin \omega / 2\left(t_{N}-d / v\right)}{\sin (\omega d / 2 v)}\right] H\left(t-t_{N-1}-\frac{L}{v}\right)
\end{aligned}
$$

and the function $P_{2}(v, t)$ in equation $(15 \mathrm{~b})$ as

$$
\begin{aligned}
P_{2}(v, t)=[ & {\left[1-\cos \omega\left(t-t_{N}\right)\right] H\left(t-t_{N}\right) } \\
& +\left\{\sum_{k=1}^{N-1}\left[\cos \omega\left(t-t_{k}-L / v\right)-\cos \omega\left(t-t_{k}\right)\right]\right\} H\left(t-t_{N-1}-L / v\right) .
\end{aligned}
$$

Through the introduction of the following relations:

$$
\begin{gathered}
\cos \omega\left(t-t_{k}-\frac{L}{v}\right)-\cos \left(t-t_{k}\right)=2 \sin \left(\frac{\omega L}{2 v}\right) \sin \left[\omega\left(t-t_{k}-\frac{L}{2 v}\right)\right], \\
\sum_{k=1}^{N-1} \sin \omega\left(t-t_{k}-\frac{L}{2 v}\right)=\sin \omega\left(t-\frac{L}{2 v}\right)+\sin \omega\left(t-\frac{t_{N}+L / v}{2}\right) \frac{\sin \omega / 2\left(t_{N}-d / v\right)}{\sin (\omega d / 2 v)},
\end{gathered}
$$

the function $P_{2}(v, t)$ in equation (17) can be rearranged as

$$
\begin{aligned}
P_{2}(v, t)= & {\left[1-\cos \omega\left(t-t_{N}\right)\right] H\left(t-t_{N}\right)+2 \sin \frac{\omega L}{2 v} } \\
& \times\left[\sin \omega\left(t-\frac{L}{2 v}\right)+\sin \omega\left(t-\frac{t_{N}+L / v}{2}\right) \frac{\sin \omega / 2\left(t_{N}-d / v\right)}{\sin (\omega d / 2 v)}\right] H\left(t-t_{N-1}-L / v\right) .
\end{aligned}
$$

By the use of equations (14), (16), and (19), the dynamic response for the midpoint of the elastically supported beam can be obtained from equation (4) as

$$
u(L / 2, t)=\frac{\Delta_{s t}(1+\kappa)}{1+4 \kappa / \pi} \times\left[Q_{1}(v, t) H\left(t-t_{N}\right)+Q_{2}(v, t) H\left(t-t_{N-1}-L / v\right)\right],
$$

where the dynamic response factors $Q_{1}(v, t)$ and $Q_{2}(v, t)$ are

$$
\begin{aligned}
Q_{1}(v, t)= & \frac{\sin \Omega\left(t-t_{N}\right)-S \sin \omega\left(t-t_{N}\right)}{1-S^{2}}+\kappa\left[1-\cos \omega\left(t-t_{N}\right)\right], \\
Q_{2}(v, t)= & 2\left[\kappa \sin (\pi / 2 S)-S \frac{\cos (\pi / 2 S)}{1-S^{2}}\right] \times\left[\sin \omega\left(t-\frac{L}{2 v}\right)\right. \\
& \left.+\sin \omega\left(t-\frac{t_{N}+L / v}{2}\right) \times \frac{\sin \omega\left[\left(t_{N}-d / v\right) / 2\right]}{\sin (\omega d / 2 v)}\right] .
\end{aligned}
$$


It should be noted that the relation $\omega L / 2 v=\pi / 2 S$ has been utilized in arriving at equation (21). From equation (20), it can be seen that the term $Q_{1}(v, t)$ is associated with $H\left(t-t_{N}\right)$, which represents the dynamic response induced by the $N$ th moving load acting on the beam, and the term $Q_{2}(v, t)$ is associated with $H\left(t-t_{N-1}-L / v\right)$, which represents the residual response induced by the $N-1$ moving loads that have passed the beam.

From equations (21b), it can be seen that the response reaches a maximum when $\sin (\omega d / 2 v)=0$. This is exactly the condition for resonance to occur. Correspondingly, the resonance speed is denoted as $v_{\text {res }}$. Note that the resonance speed $v_{\text {res }}$ as implied by the condition $\sin (\omega d / 2 v)=0$ is independent of the stiffness ratio $\kappa$. By the L'Hospital rule, the dynamic response factor for resonance $Q_{2}\left(v_{\text {res }}, t\right)$ in equation $(21 \mathrm{~b})$ can be manipulated to yield [5]

$$
Q_{2}\left(v_{\text {res }}, t\right)=2(N-1)\left[\kappa \sin \left(\pi / 2 S_{\text {res }}\right)-S_{\text {res }} \frac{\cos \left(\pi / 2 S_{\text {res }}\right)}{1-S_{\text {res }}^{2}}\right] \times \sin \omega\left(t-\frac{L}{2 v_{\text {res }}}\right),
$$

where the subscript res means resonance. The preceding equation indicates that under the condition of resonance, larger response will be induced on the beam if there are more loads passing the beam, as implied by $(N-1)$. The other observation from equation $(22)$ is that when the signs of $\sin \left(\pi / 2 S_{\text {res }}\right)$ and $\cos \left(\pi / 2 S_{\text {res }}\right)$ are different, i.e., when the vibration phases of the elastic bearings and the beam are the same, the response factor $Q_{2}\left(v_{\text {res }}, t\right)$ attains its maximum, in the sense that the response of the beam will be amplified. In contrast, when the phases of the elastic bearings and the beam are different, the response of the beam will be less severe.

On the other hand, as can be observed from equation (21b), whenever the following condition is met, that is,

$$
\kappa \sin (\pi / 2 S)-S \frac{\cos (\pi / 2 S)}{1-S^{2}}=0
$$

the residual response caused by the previous $N-1$ moving loads that have passed the beam disappears. Because of this, the condition in equation (23) has been referred to as the condition of cancellation. Under this condition, the midpoint dynamic response of the beam in equation (20) becomes

$$
u(L / 2, t)=\frac{\Delta_{s t}(1+\kappa)}{1+4 \kappa / \pi} \times\left\{\frac{\sin \Omega\left(t-t_{N}\right)-S_{c a n} \sin \omega\left(t-t_{N}\right)}{1-S_{c a n}^{2}}+\kappa\left[1-\cos \omega\left(t-t_{N}\right)\right]\right\}
$$

where the subscript can means cancellation. As can be seen, whenever the condition of cancellation is met, the response of the beam is determined solely by the $N$ th moving load, while the effect of the moving loads that have passed the beam is fully suppressed.

\section{CONSIDERATION OF THE EFFECT OF DAMPING}

Consider the case when the resonance condition implied by equation $(21 \mathrm{~b})$ is met, i.e., $\sin \left(\omega d / 2 v_{\text {res }}\right)=0$, or $\omega d / v_{\text {res }}=2 n \pi$, with $n=1,2,3 \ldots$, and when the $N$ th moving load is acting on the beam at time $t=t_{E}+t_{N}$, that is, $t=t_{E}+(N-1) d / v_{\text {res }}$, as shown in Figure 4. Then $\omega\left(t-t_{k}\right)=\omega\left(t_{E}+t_{N}-t_{k}\right)=\omega t_{E}+(N-k) \omega d / v_{\text {res }}=\omega t_{E}+2 n \pi(N-k)$ and the 


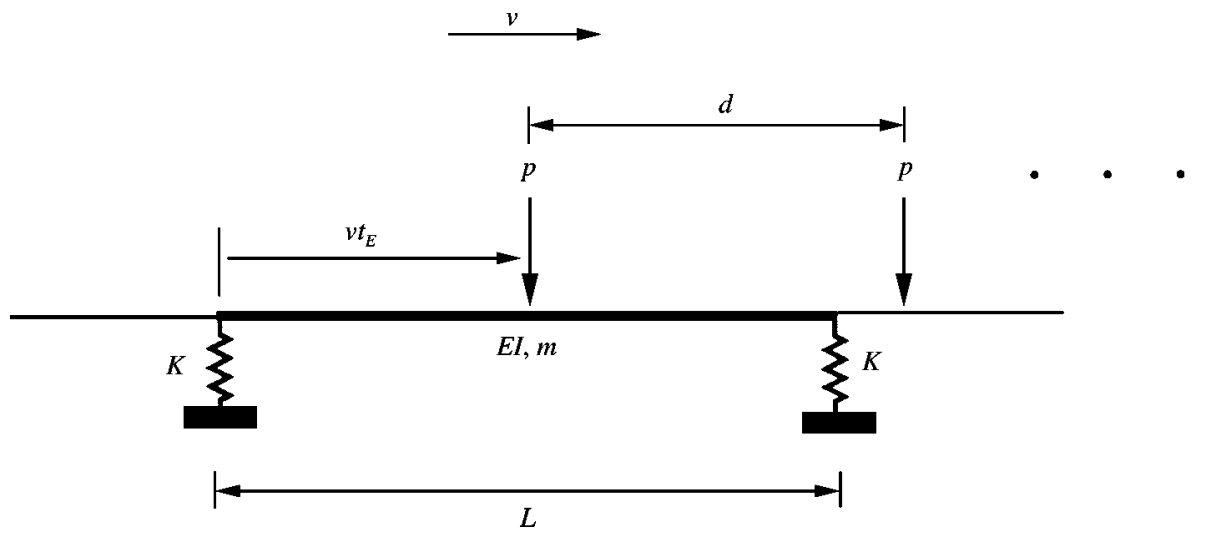

Figure 4. The $N$ th moving load is acting at position $v t_{E}$.

following relations can be derived:

$$
\begin{aligned}
& \sin \Omega\left(t-t_{k}\right)+\sin \Omega\left(t-t_{k}-L / v_{r e s}\right)=0,0<t<t_{N}, \\
& \sin \omega\left(t-t_{k}\right)=\sin \omega t_{E}, \sin \omega\left(t-t_{k}-L / v_{r e s}\right)=\sin \omega\left(t_{E}-L / v_{r e s}\right), \\
& \cos \omega\left(t-t_{k}\right)=\cos \omega t_{E}, \cos \omega\left(t-t_{k}-L / v_{\text {res }}\right)=\cos \omega\left(t_{E}-L / v_{\text {res }}\right) .
\end{aligned}
$$

By introducing the preceding relations into equation (13), where the effect of damping of the beam has been considered, the resonance response for the elastically supported beam under the action of the last (i.e., the $N$ th) moving load can be expressed as

$$
\begin{aligned}
q_{r e s}\left(t_{E}\right) \cong & \frac{\Delta_{s t}(1+\kappa)}{1+4 \kappa / \pi} \times\left\{\left[\frac{\sin \Omega t_{E}-S_{\text {res }} \mathrm{e}^{-\xi \omega t_{E}} \sin \omega t_{E}}{1-S_{\text {res }}^{2}}+\kappa\left(1-\mathrm{e}^{-\xi \omega t_{E}} \cos \omega t_{E}\right)\right] H\left(t_{E}\right)\right. \\
& +\mathrm{e}^{-\xi \omega\left(t_{E}+t_{N}\right)} \sum_{k=1}^{N-1} \mathrm{e}^{\xi \omega t_{k}} \times\left[\frac{-S_{\text {res }}}{1-S_{\text {res }}^{2}}\left(\sin \omega t_{E}+\mathrm{e}^{\xi \pi / S_{\text {res }}} \sin \omega\left(t_{E}-\frac{L}{v_{\text {res }}}\right)\right)\right. \\
& \left.\left.+\kappa\left(\mathrm{e}^{\xi \pi / S_{\text {res }}} \cos \omega\left(t_{E}-\frac{L}{v_{\text {res }}}\right)-\cos \omega t_{E}\right)\right] \times H\left(t_{E}-\frac{L-d}{v_{\text {res }}}\right)\right\} .
\end{aligned}
$$

Further, by the approximation in expansion for the exponential function, $\exp \left(\xi \pi / S_{\text {res }}\right) \cong 1+\xi \pi / S_{\text {res }}$ for $\xi \pi / S_{\text {res }} \leqslant 0 \cdot 3$, and the following relations for the series sum:

$\sum_{k=1}^{N-1} \mathrm{e}^{\xi \omega t_{k}}=\sum_{k=1}^{N} \mathrm{e}^{\frac{\xi \omega d}{v_{r e s}}(k-1)}=\frac{\mathrm{e}^{\left(\xi \omega d / v_{\text {res }}\right)(N-1)}-1}{\mathrm{e}^{\xi \omega d / v_{\text {res }}}-1}, \mathrm{e}^{-\xi \omega t_{N}} \sum_{k=1}^{N-1} \mathrm{e}^{\xi\left(\omega t_{k}\right.}=\frac{1-\mathrm{e}^{-\left(\xi \omega d / v_{\text {res }}\right)(N-1)}}{\mathrm{e}^{\xi \omega d / v_{\text {res }}}-1}$,

the dynamic response in equation (26) can further be expressed as

$$
\begin{aligned}
q_{r e s}\left(t_{E}\right) \cong & \frac{\Delta_{s t}(1+\kappa)}{1+4 \kappa / \pi} \times\left\{\left[\frac{\sin \Omega t_{E}-S_{\text {res }} \mathrm{e}^{-\xi \omega t_{k}} \sin \omega t_{E}}{1-S_{\text {res }}^{2}}+\kappa\left(1-\mathrm{e}^{-\xi \omega t_{k}} \cos \omega t_{E}\right)\right] H\left(t_{E}\right)\right. \\
& +\left[2\left(\kappa \sin \frac{\pi}{2 S_{\text {res }}}-\frac{S_{\text {res }}}{1-S_{\text {res }}^{2}} \cos \frac{\pi}{2 S_{\text {res }}}\right) \sin \omega\left(t_{E}-\frac{L}{2 v_{r e s}}\right)\right.
\end{aligned}
$$




$$
\begin{aligned}
& \left.+\frac{\xi \pi}{S_{\text {res }}}\left(\kappa \cos \omega\left(t_{E}-L / v_{\text {res }}\right)-\frac{S_{\text {res }}}{1-S_{\text {res }}^{2}} \sin \omega\left(t_{E}-L / v_{\text {res }}\right)\right)\right] \mathrm{e}^{-\xi \omega t_{E}} \\
& \left.\times \frac{1-\mathrm{e}^{-\xi(N-1) \omega d / v_{\text {res }}}}{\mathrm{e}^{\xi \omega d / v_{\text {res }}}-1} H\left(t_{E}-(L-d) / v_{\text {res }}\right)\right\} .
\end{aligned}
$$

For the special case of zero damping, i.e., by letting $\xi=0$, the dynamic response in equation (28) reduces to

$$
\begin{aligned}
q_{r e s}\left(t_{E}\right)= & \frac{\Delta_{s t}(1+\kappa)}{1+4 \kappa / \pi} \times\left\{\left[\frac{\sin \Omega t_{E}-S_{\text {res }} \sin \omega t_{E}}{1-S_{\text {res }}^{2}}+\kappa\left(1-\cos \omega t_{E}\right)\right] H\left(t_{E}\right)\right. \\
& +2(N-1)\left(\kappa \sin \frac{\pi}{2 S_{\text {res }}}-\frac{S_{\text {res }}}{1-S_{\text {res }}^{2}} \cos \frac{\pi}{2 S_{\text {res }}}\right) \sin \omega\left(t_{E}-\frac{L}{2 v_{\text {res }}}\right) \\
& \left.\times H\left(t_{E}-\frac{L-d}{v_{\text {res }}}\right)\right\} \\
= & \frac{\Delta_{\text {st }}(1+\kappa)}{1+4 \kappa / \pi}\left[Q_{1}\left(v_{\text {res }}, t_{E}\right) H\left(t-t_{N}\right)+Q_{2}\left(v_{\text {res }}, t\right) H\left(t-t_{N-1}-d / v_{\text {res }}\right)\right]
\end{aligned}
$$

which is identical to the one given in equation (20) for the condition of resonance.

For the purpose of obtaining some closed-form solutions, let us assume that there is an infinite number of moving loads crossing the beam. By letting the number of vehicles approach infinity, $N \rightarrow \infty$, and using the relation $\exp \left(\xi \omega d / v_{\text {res }}\right)-1 \cong \xi \pi d /\left(S_{\text {res }} L\right)$ for light damping, equation (28) becomes

$$
\begin{aligned}
q_{\text {res }}\left(t_{E}\right) \cong & \frac{\Delta_{s t}(1+\kappa)}{1+4 \kappa / \pi} \times\left\{\left[\frac{\sin \Omega t_{E}-S_{\text {res }} \mathrm{e}^{-\xi \omega t_{E}} \sin \omega t_{E}}{1-S_{\text {res }}^{2}}+\kappa\left(1-\mathrm{e}^{-\xi \omega t_{E}} \cos \omega t_{E}\right)\right] H\left(t_{E}\right)\right. \\
& +\mathrm{e}^{-\xi \omega t_{E}} \times \frac{S_{\text {res }} L}{\xi \pi d}\left[2\left(\kappa \sin \frac{\pi}{2 S_{\text {res }}}-\frac{S_{\text {res }}}{1-S_{\text {res }}^{2}} \cos \frac{\pi}{2 S_{\text {res }}}\right) \sin \left(\omega t_{E}-\frac{\pi}{2 S_{\text {res }}}\right)\right. \\
& \left.\left.+\frac{\xi \pi}{S_{\text {res }}}\left(\kappa \cos \left(\omega t_{E}-\frac{\pi}{S_{\text {res }}}\right)-\frac{S_{\text {res }}}{1-S_{\text {res }}^{2}} \sin \left(\omega t_{E}-\frac{\pi}{S_{\text {res }}}\right)\right)\right] H\left(t_{E}-\frac{L-d}{v_{\text {res }}}\right)\right\} .
\end{aligned}
$$

As can be verified from equation (30) and in the examples to follow, if the effect of damping is considered, the resonant response of the beam subjected to an infinite number of moving loads remains more or less constant. Further, by the use of the following relations:

$$
\begin{aligned}
& \cos \left(\omega t_{E}-\frac{\pi}{S_{\text {res }}}\right)=\cos \frac{\pi}{2 S_{\text {res }}} \cos \left(\omega t_{E}-\frac{\pi}{2 S_{\text {res }}}\right)+\sin \frac{\pi}{2 S_{\text {res }}} \sin \left(\omega t_{E}-\frac{\pi}{2 S_{\text {res }}}\right), \\
& \sin \left(\omega t_{E}-\frac{\pi}{S_{\text {res }}}\right)=\cos \frac{\pi}{2 S_{\text {res }}} \sin \left(\omega t_{E}-\frac{\pi}{2 S_{\text {res }}}\right)-\sin \frac{\pi}{2 S_{\text {res }}} \cos \left(\omega t_{E}-\frac{\pi}{2 S_{\text {res }}}\right),
\end{aligned}
$$


the resonance response in equation (30) becomes

$$
\begin{aligned}
q_{r e s}\left(t_{E}\right) \cong & \frac{\Delta_{s t}(1+\kappa)}{1+4 \kappa / \pi} \times\left\{\left[\frac{\sin \Omega t_{E}-S_{r e s} \mathrm{e}^{-\xi \omega t_{E}} \sin \omega t_{E}}{1-S_{r e s}^{2}}+\kappa\left(1-\mathrm{e}^{-\xi \omega t_{E}} \cos \omega t_{E}\right)\right] H\left(t_{E}\right)\right. \\
& +\frac{L}{d} \mathrm{e}^{-\xi \omega t_{E}} \times\left[\left(\frac{2 S_{\text {res }}}{\xi \pi}+1\right)\left(\kappa \sin \frac{\pi}{2 S_{r e s}}-\frac{S_{\text {res }}}{1-S_{\text {res }}^{2}} \cos \frac{\pi}{2 S_{\text {res }}}\right) \sin \left(\omega t_{E}-\frac{\pi}{2 S_{\text {res }}}\right)\right. \\
& \left.\left.+\left(\kappa \cos \frac{\pi}{2 S_{\text {res }}}+\frac{S_{\text {res }}}{1-S_{\text {res }}^{2}} \sin \frac{\pi}{2 S_{\text {res }}}\right) \cos \left(\omega t_{E}-\frac{\pi}{2 S_{r e s}}\right)\right] H\left(t_{E}-\frac{L-d}{v_{\text {res }}}\right)\right\}
\end{aligned}
$$

Since $\sin \left(\omega t_{E}-\pi / 2 S_{\text {res }}\right)$ and $\cos \left(\omega t_{E}-\pi / 2 S_{\text {res }}\right)$ are out of phase, when the function $\sin \left(\omega t_{E}-\pi / 2 S_{\text {res }}\right)$ reaches the maximum, the function $\cos \left(\omega t_{E}-\pi / 2 S_{\text {res }}\right)$ is at its minimum. As far as the maximum response is concerned, the preceding expression can be approximated by dropping the term in the third line as follows:

$$
\begin{aligned}
& q_{\text {res }, \text { max }}\left(t_{E}\right) \cong \frac{\Delta_{s t}(1+\kappa)}{1+4 \kappa / \pi} \times\left\{\left[\frac{\sin \Omega t_{E}-S_{\text {res }} \mathrm{e}^{-\xi \omega t_{E}} \sin \omega t_{E}}{1-S_{r e s}^{2}}+\kappa\left(1-\mathrm{e}^{-\xi \omega t_{E}} \cos \omega t_{E}\right)\right] H\left(t_{E}\right)\right. \\
& \left.+\frac{L}{d} \mathrm{e}^{-\xi \omega t_{E}}\left(\frac{2 S_{r e s}}{\xi \pi}+1\right)\left(\kappa \sin \frac{\pi}{2 S_{\text {res }}}-\frac{S_{\text {res }}}{1-S_{\text {res }}^{2}} \cos \frac{\pi}{2 S_{\text {res }}}\right) \sin \left(\omega t_{E}-\frac{\pi}{2 S_{\text {res }}}\right) H\left(t_{E}-\frac{L-d}{v_{\text {res }}}\right)\right\},
\end{aligned}
$$

where it is recognized that $2 S_{\text {res }} / \xi \pi+1>1$. At this point, we have derived the maximum response for the elastically supported beam in equation (33) considering the effect of damping.

\section{ENVELOPE FORMULA FOR RESONANT RESPONSE}

In this section, envelope formulas will be derived for the elastically supported beam based on the maximum response presented in equation (33), assuming that the beam is lightly damped and is subjected to an infinite number of moving loads. When the condition of resonance is met, while the condition of cancellation as given in equation (23) is not, the dynamic response of the beam is dominated by the term containing $H\left(t_{E}-(L-d) / v_{\text {res }}\right)$ in equation (33). Moreover, the function $\sin \left(\omega t_{E}-\pi / 2 S_{\text {res }}\right)$ reaches its maximum when

$$
\omega t_{E}=\frac{\pi}{2}+\frac{\pi}{2 S_{r e s}}=\frac{\pi\left(1+S_{r e s}\right)}{2 S_{r e s}} .
$$

Substituting equation (34) into equation (33) and noting that $\exp \left(-\xi \omega t_{E}\right) \cong 1$ for light damping, one obtains

$$
\begin{aligned}
& q_{\text {res, } \max }\left(t_{E}\right) \cong \frac{\Delta_{s t}(1+\kappa)}{1+4 \kappa / \pi} \times\left\{\left[\kappa+\frac{\cos \left(S_{\text {res }} \pi / 2\right)}{1-S_{\text {res }}^{2}}+\left(\kappa \sin \frac{\pi}{2 S_{\text {res }}}-\frac{S_{\text {res }}}{1-S_{\text {res }}^{2}} \cos \frac{\pi}{2 S_{\text {res }}}\right) \mathrm{e}^{-\xi \pi\left(1+S_{\text {res }}\right) / 2 S_{\text {res }}}\right] H\left(t_{E}\right)\right. \\
& \left.+\frac{L}{d}\left(2 \frac{S_{\text {res }}}{\xi \pi}+1\right)\left(\kappa \sin \frac{\pi}{2 S_{\text {res }}}-\frac{S_{\text {res }}}{1-S_{\text {res }}^{2}} \cos \frac{\pi}{2 S_{\text {res }}}\right) \mathrm{e}^{-\xi \pi\left(1+S_{\text {res }}\right) / 2 S_{\text {res }}} H\left(t_{E}-\frac{L-d}{v_{\text {res }}}\right)\right\} .
\end{aligned}
$$


Accordingly, the absolute maximum response is

$$
\begin{aligned}
q_{\text {res }, \text { max }} \approx & \frac{\Delta_{s t}(1+\kappa)}{1+4 \kappa / \pi} \times\left\{\left[\kappa+\frac{\left|\cos \left(S_{\text {res }} \pi / 2\right)\right|}{1-S_{\text {res }}^{2}}\right]\right. \\
& \left.+\left|\kappa \sin \frac{\pi}{2 S_{\text {res }}}-\frac{S_{\text {res }}}{1-S_{\text {res }}^{2}} \cos \frac{\pi}{2 S_{\text {res }}}\right|\left[\frac{L}{d}\left(\frac{2 S_{\text {res }}}{\xi \pi}+1\right)+1\right] \mathrm{e}^{-\xi \pi\left(1+S_{\text {res }} / 2 S_{\text {res }}\right.}\right\} .
\end{aligned}
$$

For the case of light damping considered in this study, $\xi \pi / S_{\text {res }}<0 \cdot 3$, the following relations may be adopted:

$$
\begin{gathered}
\frac{\cos \left(S_{\text {res }} \pi / 2\right)}{1-S_{\text {res }}^{2}} \cong 1,1-S_{\text {res }}^{2} \cong 1, \\
{\left[\frac{L}{d}\left(\frac{2 S_{\text {res }}}{\xi \pi}+1\right)+1\right] \mathrm{e}^{-\xi \pi\left(1+S_{\text {res }}\right) / 2 S_{\text {res }}} \cong \frac{L}{d} \times \frac{2 S_{\text {res }}}{\xi \pi} .}
\end{gathered}
$$

It follows that the maximum response in equation (36) reduces to

$$
q_{\text {res,max }} \approx \frac{\Delta_{s t}(1+\kappa)}{1+4 \kappa / \pi} \times\left(1+\kappa+\frac{L}{d} \frac{2 S_{r e s}}{\xi \pi}\left|\kappa \sin \frac{\pi}{2 S_{r e s}}-S_{\text {res }} \cos \frac{\pi}{2 S_{\text {res }}}\right|\right)
$$

which is applicable for the case where the resonance condition is met, but the cancellation condition is not.

On the other hand, when both the resonance and cancellation conditions given in equation (23) are satisfied, i.e., $S_{r e s}=S_{c a n}$, the response in equation (33) becomes

$$
q_{r e s}\left(t_{E}\right) \cong \frac{\Delta_{s t}(1+\kappa)}{1+4 \kappa / \pi} \times\left[\frac{\sin \Omega t_{E}-S_{c a n} \mathrm{e}^{-\xi \omega t_{E}} \sin \omega t_{E}}{1-S_{c a n}^{2}}+\kappa\left(1-\mathrm{e}^{-\xi \omega t_{E}} \cos \omega t_{E}\right)\right] H\left(t_{E}\right) .
$$

Here, due to the damping effect and consideration of operating speeds in the range $0<S_{\text {res }}<0 \cdot 3$, the forced vibration term $\sin \Omega t_{E}$ and the constant $\kappa$ will dominate the response. By letting $\sin \Omega t_{E}=1$, or $\omega t_{E}=\pi /\left(2 S_{\text {res }}\right)$, equation (39) becomes

$$
q_{r e s}\left(t_{E}\right) \cong \frac{\Delta_{s t}(1+\kappa)}{1+4 \kappa / \pi} \times\left[\frac{1}{1-S_{r e s}^{2}}+\kappa-\mathrm{e}^{-\xi \pi / 2 S_{r e s}}\left(\frac{S_{c a n} \sin \left(\pi / 2 S_{c a n}\right)}{1-S_{c a n}^{2}}-\kappa \cos \frac{\pi}{2 S_{c a n}}\right)\right] H\left(t_{E}\right)
$$

which can further be expressed as follows:

$$
q_{\text {res }, \text { max }} \approx \frac{\Delta_{s t}(1+\kappa)}{1+4 \kappa / \pi} \times\left[\kappa+\frac{1}{1-S_{c a n}^{2}}+\left(1-\frac{\xi \pi}{2 S_{c a n}}\right) \sqrt{S_{c a n}^{2}+\kappa^{2}}\right]
$$

if use is made of the relations $\exp \left(-\xi \pi / 2 S_{c a n}\right) \cong 1-\xi \pi / 2 S_{c a n}$ and $S_{c a n} /\left(1-S_{c a n}^{2}\right) \cong S_{c a n}$. This formula is valid for the case when both the condition of resonance and the condition of cancellation are satisfied. 


\section{IMPACT FACTOR AND ENVELOPE IMPACT FORMULA}

The impact factor for the deflection of an elastically supported beam subjected to the moving loads is defined as

$$
I=\frac{R_{d}(x)-R_{s}(x)}{R_{s}(x)},
$$

where $R_{d}(x)$ and $R_{s}(x)$, respectively, denote the maximum dynamic and static responses of the beam at position $x$ due to the action of the moving loads. The following is the maximum static deflection for an elastically supported beam subjected a lumped load $p$ at the midpoint:

$$
R_{s}(x)=\frac{\Delta_{s t}(1+\kappa)^{2}}{1+4 \kappa / \pi},
$$

where $\Delta_{s t}=2 p L^{3} /\left(\pi^{4} E I\right) \approx p L^{3} /(48 E I)=$ the maximum midpoint static deflection of the simple beam. By the use of equations (38), (41), and (42), the deflection impact formula for the elastically supported beam subjected to a sequence of moving loads can be expressed as

$$
\begin{aligned}
I & \approx \frac{1}{(1+\kappa)}\left[1+\kappa+\frac{L}{d} \frac{2 S_{r e s}}{\xi \pi}\left|\kappa \sin \left(\pi / 2 S_{r e s}\right)-S_{r e s} \cos \left(\pi / 2 S_{r e s}\right)\right|\right]-1 \\
& \approx \frac{L}{(1+\kappa) d} \frac{2 S_{r e s}}{\xi \pi}\left|\kappa \sin \left(\pi / 2 S_{r e s}\right)-S_{r e s} \cos \left(\pi / 2 S_{r e s}\right)\right|
\end{aligned}
$$

for the case of resonance, and

$$
\begin{aligned}
I & \approx \frac{1}{(1+\kappa)}\left[\kappa+\frac{1}{1-S_{c a n}^{2}}+\left(S_{c a n}-\frac{\xi \pi}{2}\right) \sqrt{1+\left(\frac{\kappa}{S_{c a n}}\right)^{2}}\right]-1 \\
& \approx \frac{1}{(1+\kappa)}\left[\frac{S_{c a n}^{2}}{1-S_{c a n}^{2}}+\left(S_{c a n}-\frac{\xi \pi}{2}\right) \sqrt{1+\left(\frac{\kappa}{S_{c a n}}\right)^{2}}\right]
\end{aligned}
$$

for the case when the conditions of both resonance and cancellation are met.

\section{NUMERICAL EXAMPLES}

\subsection{PHENOMENON OF RESONANCE}

As shown in Table 1, two bridges supported by elastic bearings are considered in this example. The train moving over the bridge is assumed to have $N=8$ cars each of length $d=25 \mathrm{~m}$. The weight of each car is represented by a moving load of $p=300 \mathrm{kN}$. Different values of resonant speeds computed for the two beams have been listed in Table 2, with those for the corresponding beams with hinged supports. Evidently, most of the resonance speeds can be encountered by modern high-speed trains in commercial operation. The responses of the beam traversed by the moving loads have been plotted in Figures 5-7, along with those obtained by independent finite element analyses. As can be seen, all the solutions obtained by the present approach agree excellently with the finite element 
TABLE 1

Properties of bridges

\begin{tabular}{lcclcc}
\hline$L(\mathrm{~m})$ & $m(\mathrm{t} / \mathrm{m})$ & $E I\left(k N-\mathrm{m}^{2}\right)$ & & $\omega_{0}(\mathrm{rad} / \mathrm{s})$ & $\omega(\mathrm{rad} / \mathrm{s})$ \\
\hline 23 & 30 & $1 \cdot 4 \times 10^{8}$ & $0 \cdot 24$ & $40 \cdot 3$ & $35 \cdot 1$ \\
27 & 32 & $2 \cdot 0 \times 10^{8}$ & $0 \cdot 2$ & $33 \cdot 9$ & $29 \cdot 9$ \\
\hline
\end{tabular}

TABLE 2

Resonant speeds

\begin{tabular}{lccc}
\hline $\begin{array}{l}\text { Resonant speed } \\
v_{\text {res }}=(d / 2) / n\end{array}$ & $\begin{array}{c}L=23 \mathrm{~m} \\
(n=2)\end{array}$ & $\begin{array}{c}L=27 \mathrm{~m} \\
(n=2)\end{array}$ & $\begin{array}{c}L=27 \mathrm{~m} \\
(n=4)\end{array}$ \\
\hline $\begin{array}{l}\text { Simple beam } v_{0} \\
{\left[S_{\text {res }}=d /(2 n L)\right]}\end{array}$ & $80 \mathrm{~ms}(=288 \mathrm{~km} / \mathrm{h})$ & $67 \mathrm{~m} / \mathrm{s}(=242 \mathrm{~km} / \mathrm{h})$ & $34 \mathrm{~m} / \mathrm{s}(=122 \mathrm{~km} / \mathrm{h})$ \\
Elast. supp. beam $v$ & $70 \mathrm{~m} / \mathrm{s}(=252 \mathrm{~km} / \mathrm{h})$ & $59 \mathrm{~m} / \mathrm{s}(=214 \mathrm{~km} / \mathrm{h})$ & $30 \mathrm{~m} / \mathrm{s}(=108 \mathrm{~km} / \mathrm{h})$ \\
{$\left[S_{\text {res }}=d /(2 n L)\right]$} & {$\left[S_{\text {res }}=0 \cdot 272\right]$} & {$\left[S_{\text {res }}=0 \cdot 231\right]$} & $\left(S_{\text {res }}=0 \cdot 116\right)$ \\
\hline
\end{tabular}

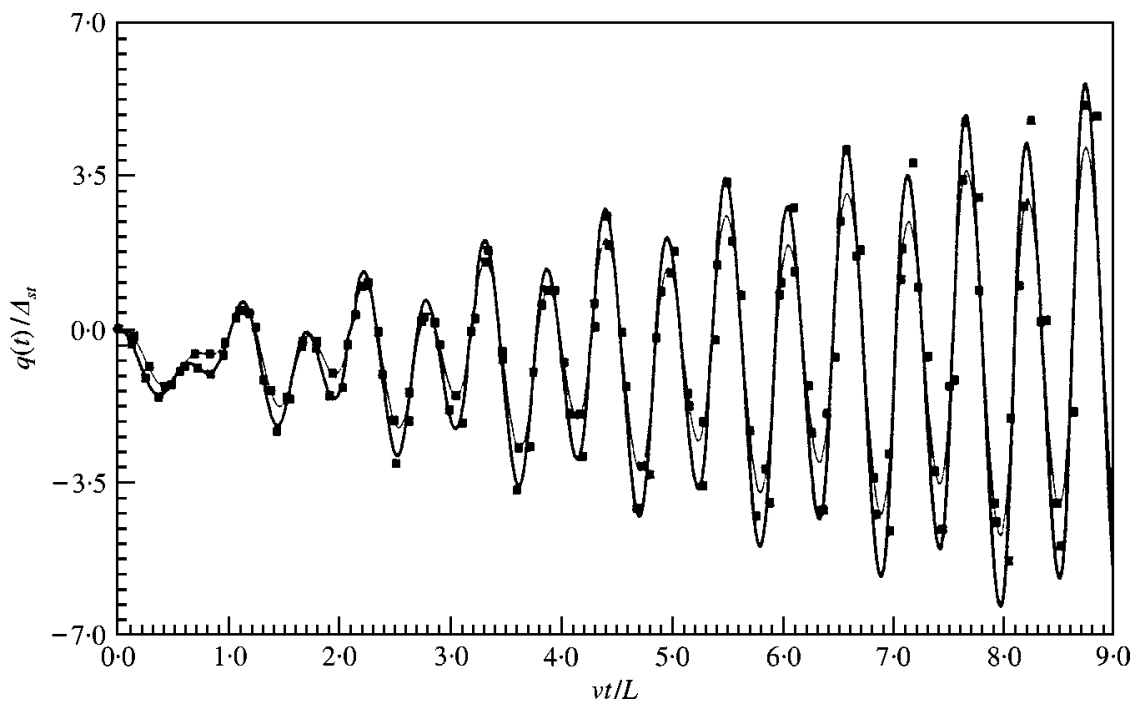

Figure 5. Time history responses at resonant speed $S=d / 4 L$ for $23 \mathrm{~m}$ beam. — - Simple beam (analytic); Elastically supported beam (analytic); $\mathbf{-}$, Finite element solutions. Span length $=23 \mathrm{~m}\left(S_{\text {res }}=0.272\right)$.

solutions. For the case when the motions of the simple beam and elastic bearings are in phase, the elastic bearings inserted at the supports can significantly increase the dynamic response of the bridge to the moving loads, as can be seen from Figures 5 and 7. Such a phenomenon is harmful to the riding comfort or maneuverability of the train. However, for the case when the motions of the simple beam and elastic bearings are out of phase, as indicated in Figure 6, the dynamic response of the beam is reduced through the introduction of the elastic bearings. 


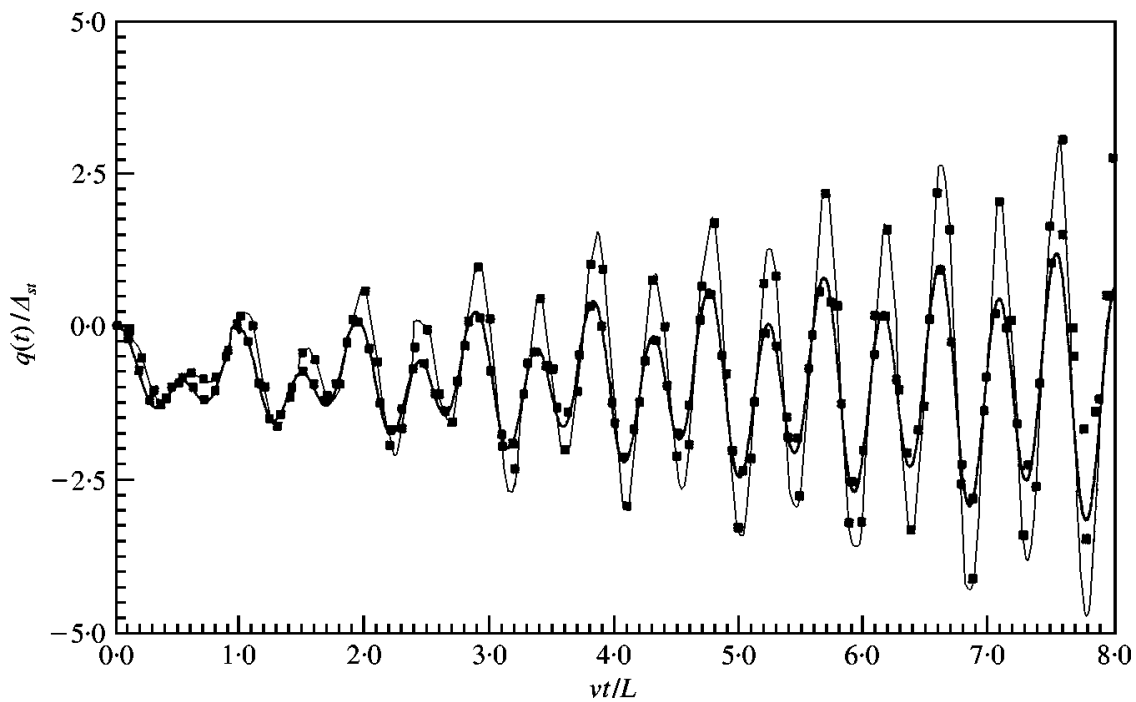

Figure 6. Time history responses at resonant speed $S=d / 4 L$ for $27 \mathrm{~m}$ beam. —— - Simple beam (analytic); — Elastically supported beam (analytic); $\mathbf{\square}$, Finite element solutions. Span length $=27 \mathrm{~m}\left(S_{\text {res }}=0.231\right)$.

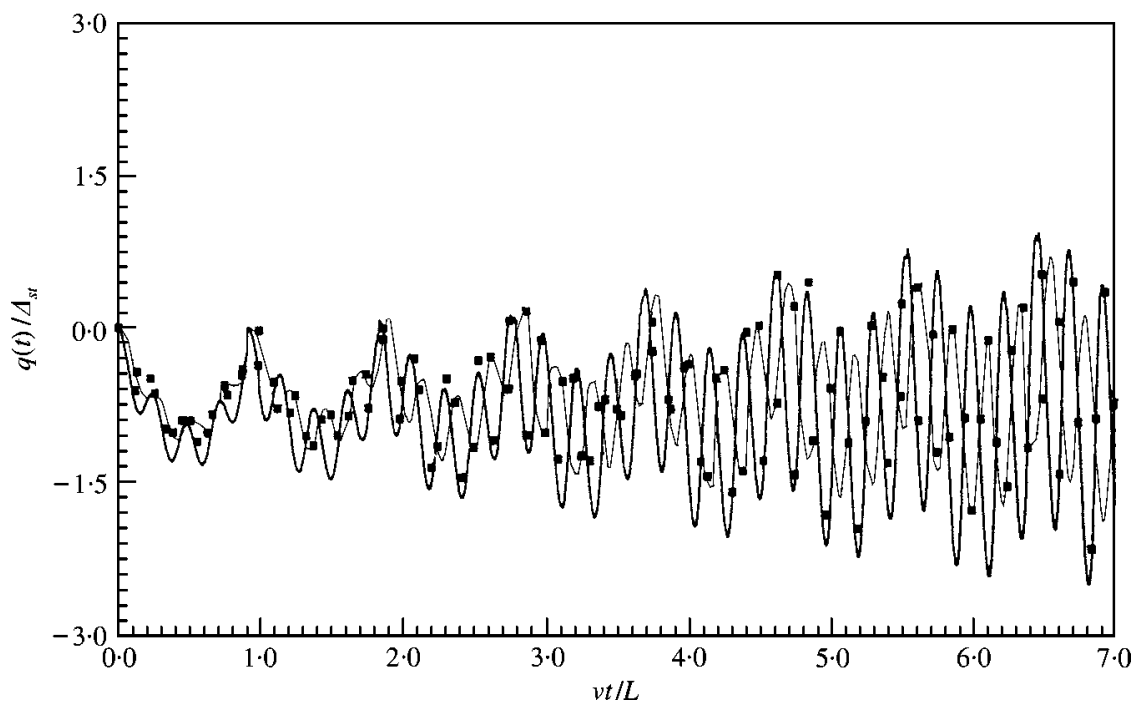

Figure 7. Time history responses at resonant speed $S=d / 8 L$ for $27 \mathrm{~m}$ beam. ——, Simple beam (analytic); — Elastically supported beam (analytic); $\mathbf{\square}$, Finite element solutions. Span length $=27 \mathrm{~m}\left(S_{\text {res }}=0.116\right)$.

\subsection{EFFECT OF DAMPING}

To investigate the effect of damping on the resonant response of an elastically supported beam due to an infinite series of moving loads, 30 moving loads are considered in this example. A damping ratio of two per cent is assumed for the beam. As can be seen from Figures 8-11, due to the presence of damping, the vibration of the beam remains rather bounded, in a steady state manner, even under the condition of resonance. This is very 


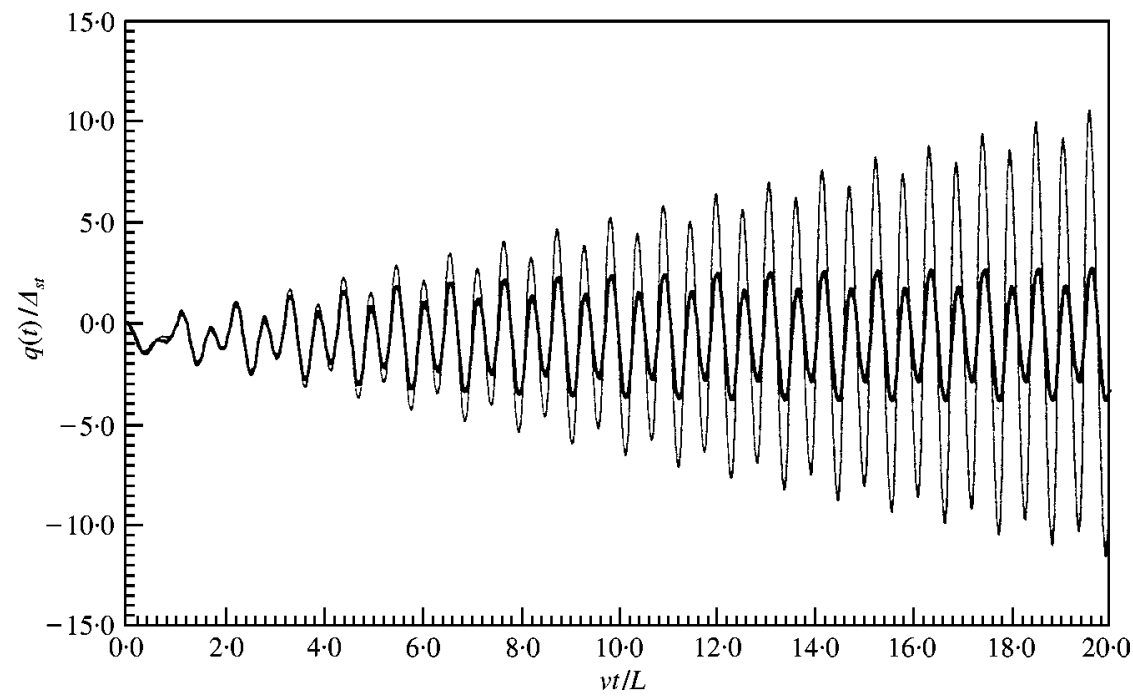

Figure 8. Comparison of time history responses at resonant speed $S=d / 4 L$ for $23 \mathrm{~m}$ simple beam. - Undamped system; - - Damped system $(\xi=0 \cdot 02)$. Span length $=23 \mathrm{~m}\left(S_{\text {res }}=0.272\right)$.

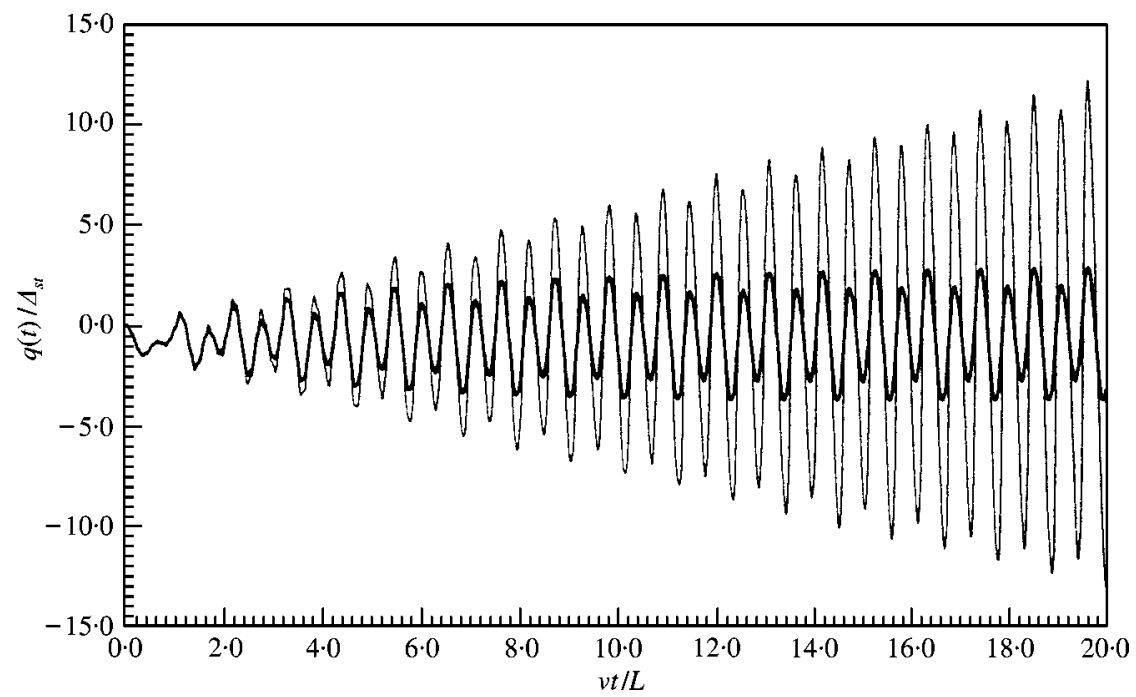

Figure 9. Comparison of time history responses at resonant speed $S=d / 4 L$ for $23 \mathrm{~m}$ elastically supported beam. ——, Undamped system; ——, Damped system $(\xi=0.02)$. Span length $=23 \mathrm{~m}\left(S_{\text {res }}=0.272\right)$.

different from the undamped case, in which the response amplitude tends to grow increasingly, as there are more loads passing the beam when the condition of resonance is reached.

\subsection{ENVELOPE IMPACT FORMULA}

Under the condition of resonance, $\sin \left(\omega d / 2 v_{\text {res }}\right)=0$, from which the resonance speed can be solved: $v_{\text {res }}=\omega d / 2 n \pi$ or $S_{\text {res }}=d / 2 n L$, with $n=1,2,3, \ldots$ Thus, the larger values 


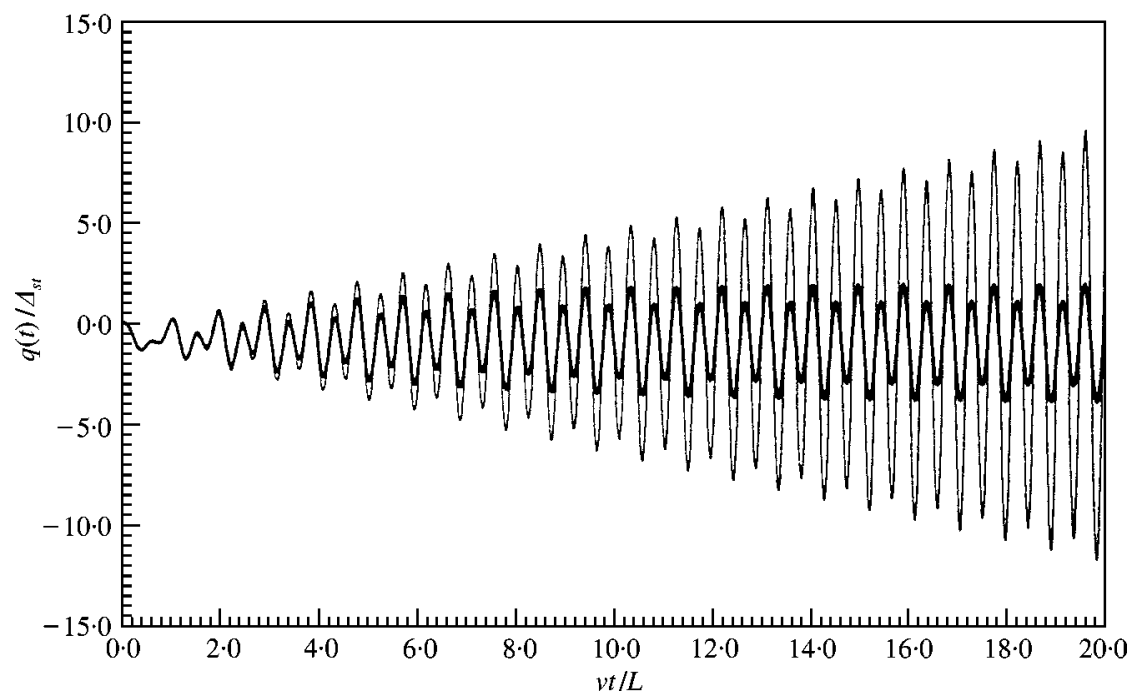

Figure 10. Comparison of time history responses at resonant speed $S=d / 4 L$ for $27 \mathrm{~m}$ simple beam. - Undamped system; —- Damped system $(\xi=0 \cdot 02)$. Span length $=27 \mathrm{~m}\left(S_{\text {res }}=0.231\right)$.

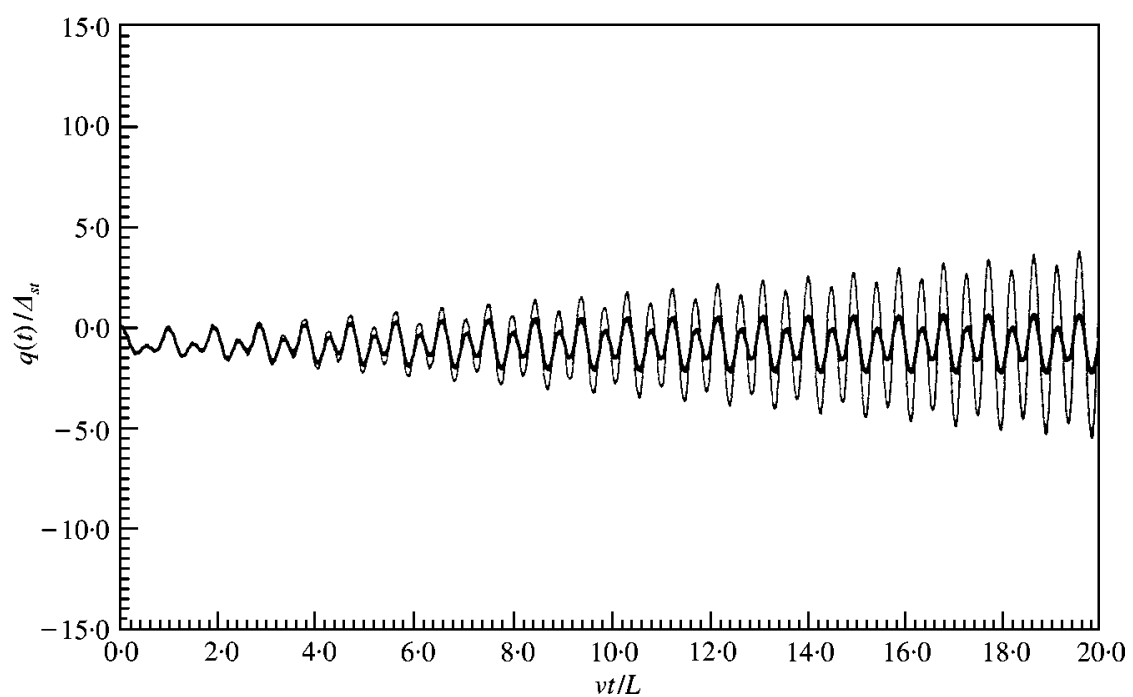

Figure 11. Comparison of time history responses at resonant speed $S=d / 4 L$ for $27 \mathrm{~m}$ elastically supported beam. - - Undamped system; —- Damped system $(\xi=0.02)$. Span length $=27 \mathrm{~m}\left(S_{\text {res }}=0.231\right)$.

computed from the envelope impact formulas (44) and (45) for all the resonance points can be connected in a piecewise sense as shown in Figures 12-15, where two values of damping ratios, $\xi=0 \cdot 02,0 \cdot 04$, are considered. The results for the simple beams are plotted in Figures 12 and 14, and those for the elastically supported beams in Figures 13 and 15. Also plotted in the figures are the impact factors $I$ computed using the more accurate equations available in section 6. The figures shown here are known as the $I-S$ (impact factor versus speed 


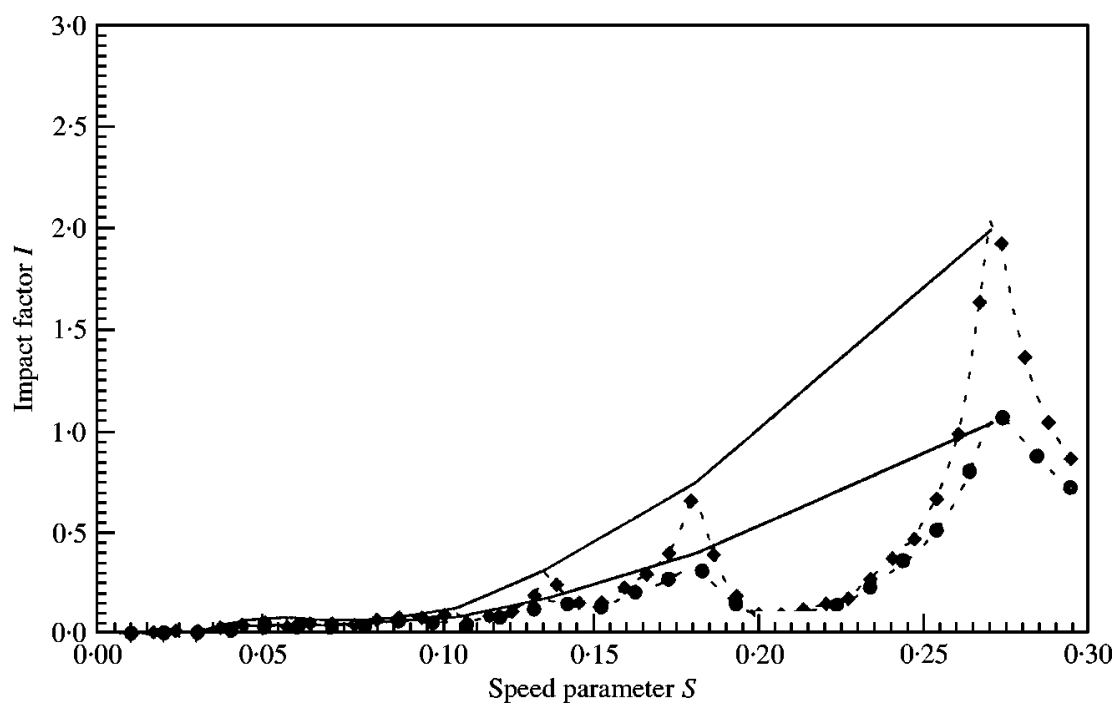

Figure 12. $I-S$ plot for $23 \mathrm{~m}$ simple beam. $-\prec-, \xi=0.02 ;--\bullet-, \xi=0 \cdot 04 ;-$, Impact formula. Span length $=23 \mathrm{~m}$.

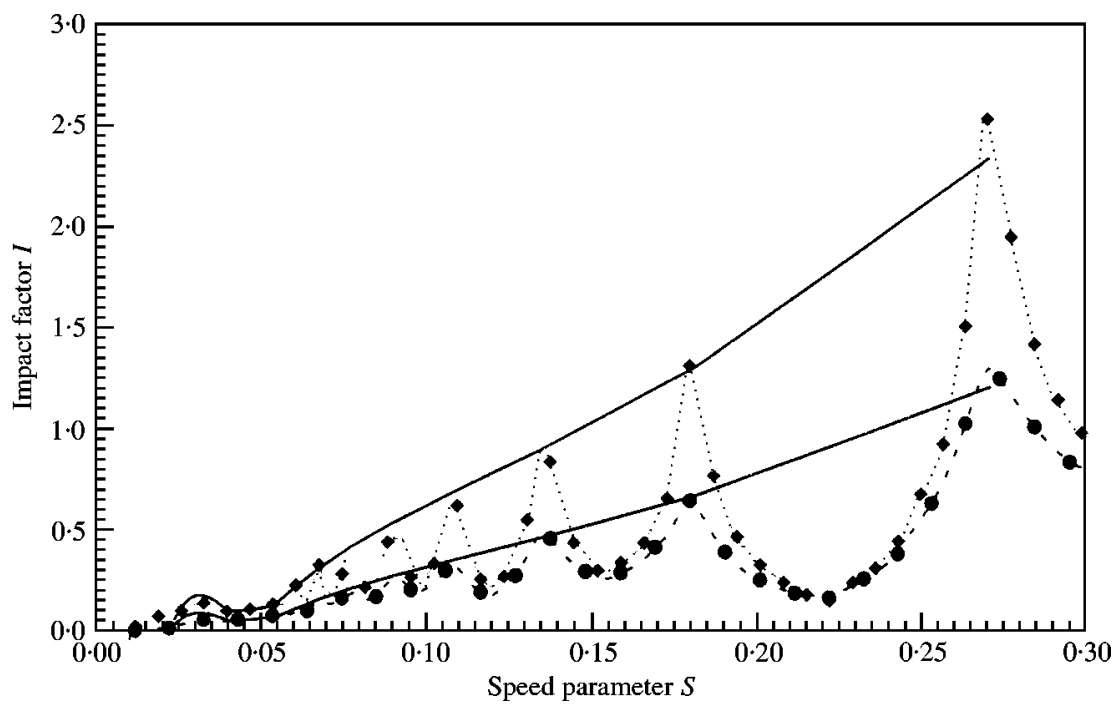

Figure 13. $I-S$ plot for $23 \mathrm{~m}$ elastically supported beam. -,$-- \xi=0.02 ;--\mathbf{-}--, \xi=0 \cdot 04 ;-$, Impact formula. Span length $=23 \mathrm{~m}$.

parameter) plots. As can be seen, the envelope impact formulas show a trend in good consistency with the more accurate $I-S$ plots for the two values of damping ratios throughout the entire range of speed parameters considered. An observation from Figures 12 and 13 for the $23 \mathrm{~m}$ beams is that the installation of elastic bearings at the supports tends to increase drastically the impact response of the beams throughout the entire range of speeds considered. On the other hand, a comparison of Figures 14 with 15 for the $27 \mathrm{~m}$ 


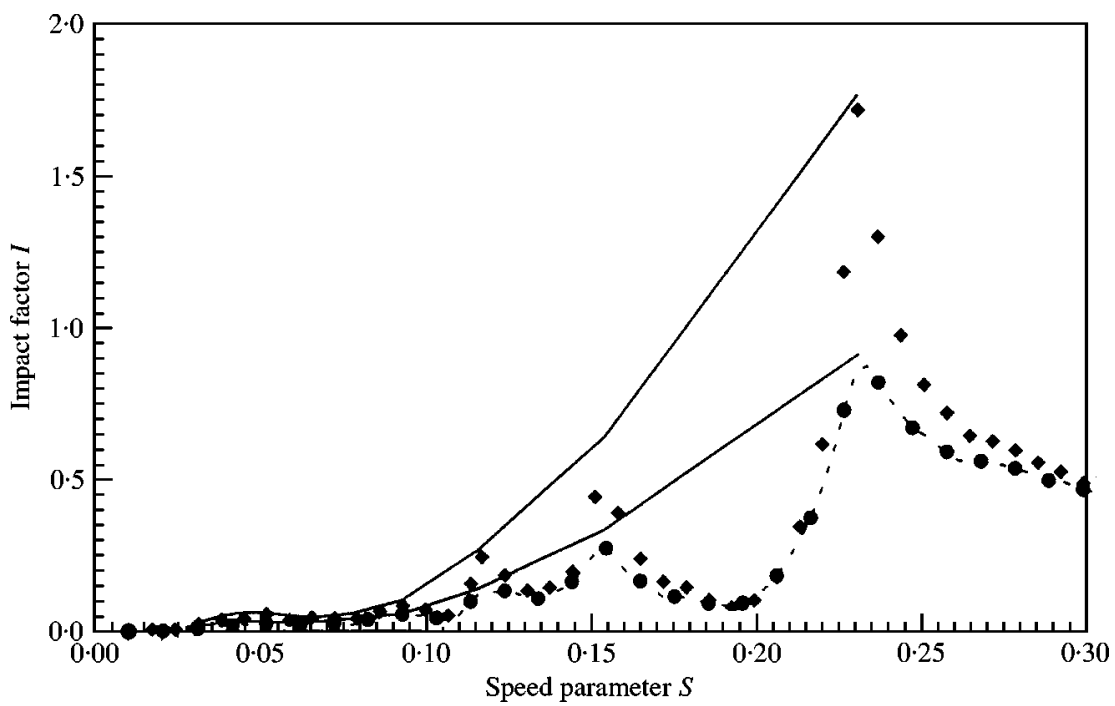

Figure 14. $I-S$ plot for $27 \mathrm{~m}$ simple beam. $-\prec-, \xi=0.02 ;--\bullet-, \xi=0.04 ;-$, Impact formula. Span length $=27 \mathrm{~m}$.

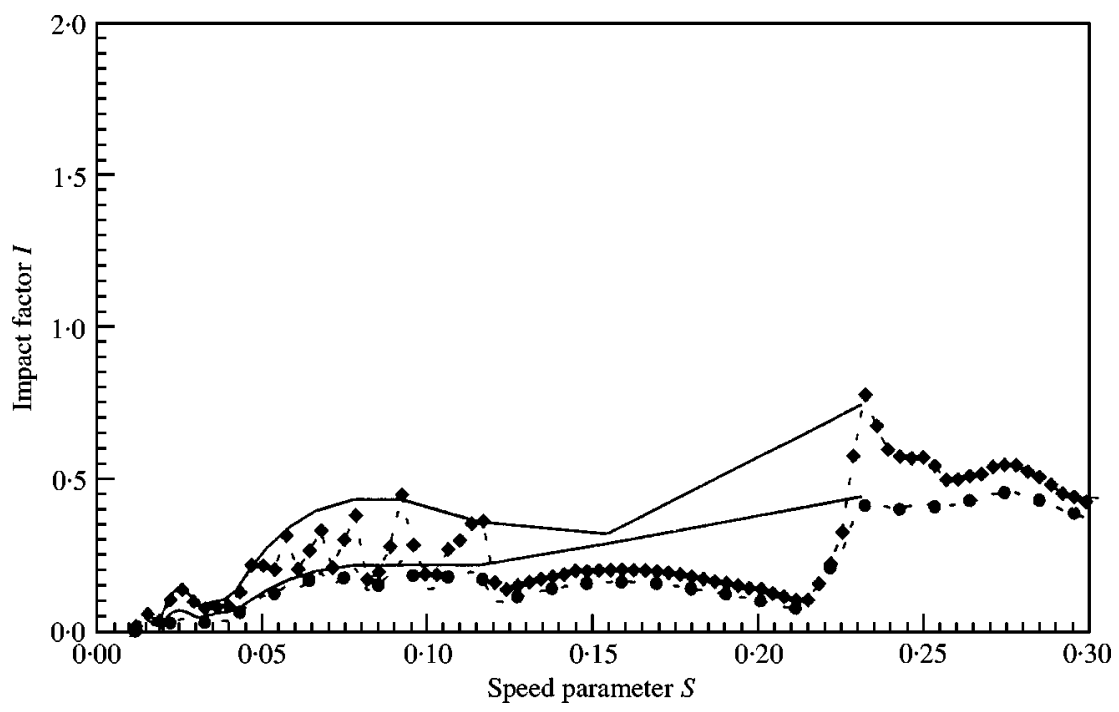

Figure 15. $I-S$ plot for $27 \mathrm{~m}$ elastically supported beam. $-\prec-, \xi=0.02 ;--\mathbf{-}-, \quad \xi=0.04 ;-$, Impact formula. Span length $=27 \mathrm{~m}$.

beams indicates that the installation of elastic bearings may amplify the impact response only over the low speed range $(S<0.125)$, but may suppress the response for the high-speed range $(S>0 \cdot 125)$. In particular, the three-dimensional $I-S-L / d$ plot for the impact factor has been drawn in Figures 16 and 17 for two values of stiffness ratios, $\kappa=0 \cdot 15,0 \cdot 25$. Evidently, the use of less rigid elastic bearings, as implied by a larger $\kappa$, tends to increase the impact response of elastically supported beams. 


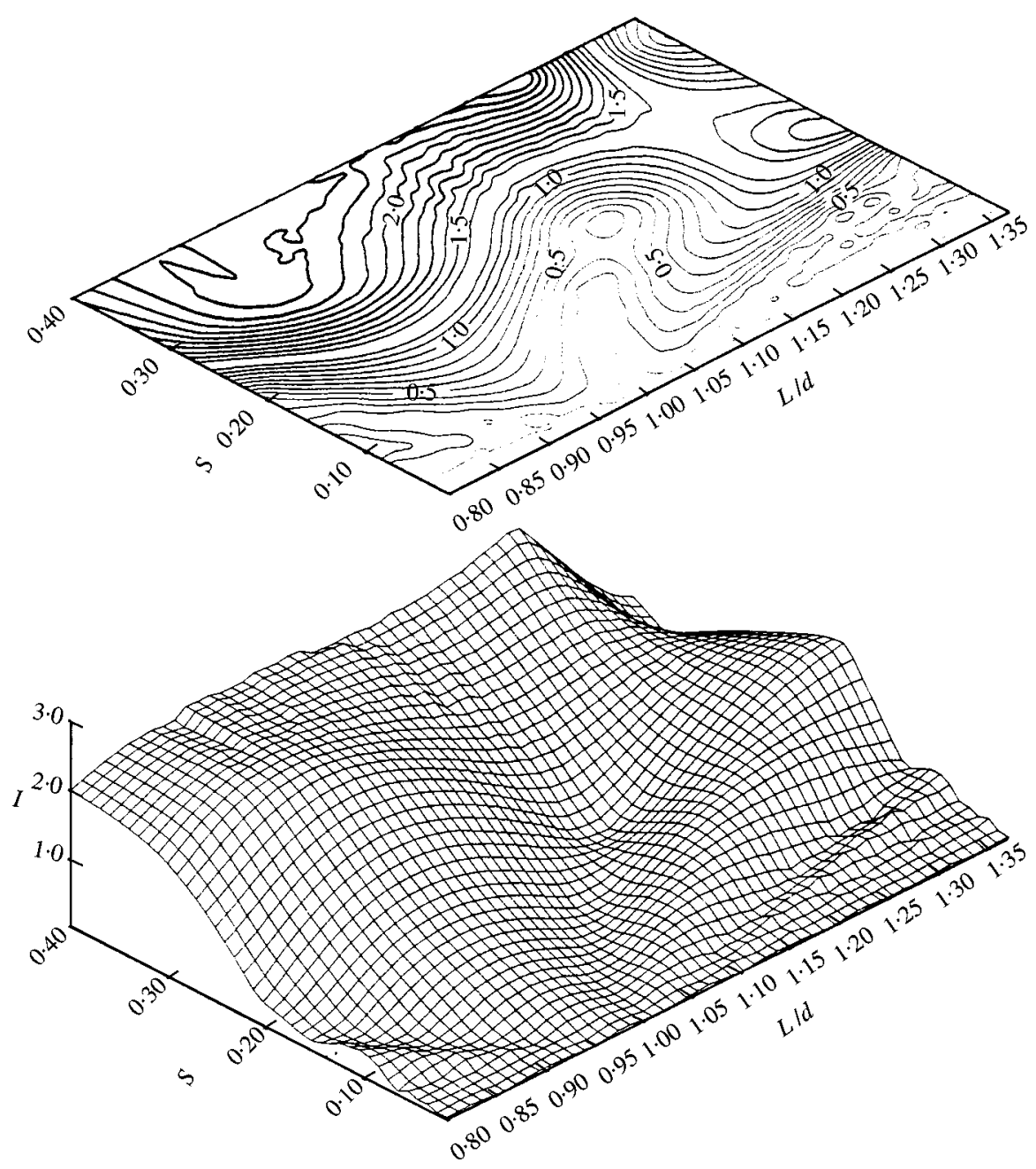

Figure 16. $I-S-L / d$ Diagram for $\xi=0 \cdot 02, \kappa=0 \cdot 15$.

\section{CONCLUSIONS}

In this study, the impact response of elastically supported beams subjected to a sequence of moving loads has been investigated by an analytical approach. Light damping is assumed for the beams. The conditions of both resonance and cancellation are identified. Unlike the case for the undamped beam, whose resonance response tends to grow as there are more moving loads passing the beam, the resonance response for the damped beam remains more or less constant regardless of the number of moving loads that have passed the beam. For the case of an infinite number of moving loads, an envelope impact formula is derived for the elastically supported beam with light damping. It is concluded that the installation of elastic bearings may increase the response of the beam under most resonance conditions. The more flexible the elastic bearings, the larger the response of the beam is. Future field tests will be considered to experimentally verify the results presented herein. 


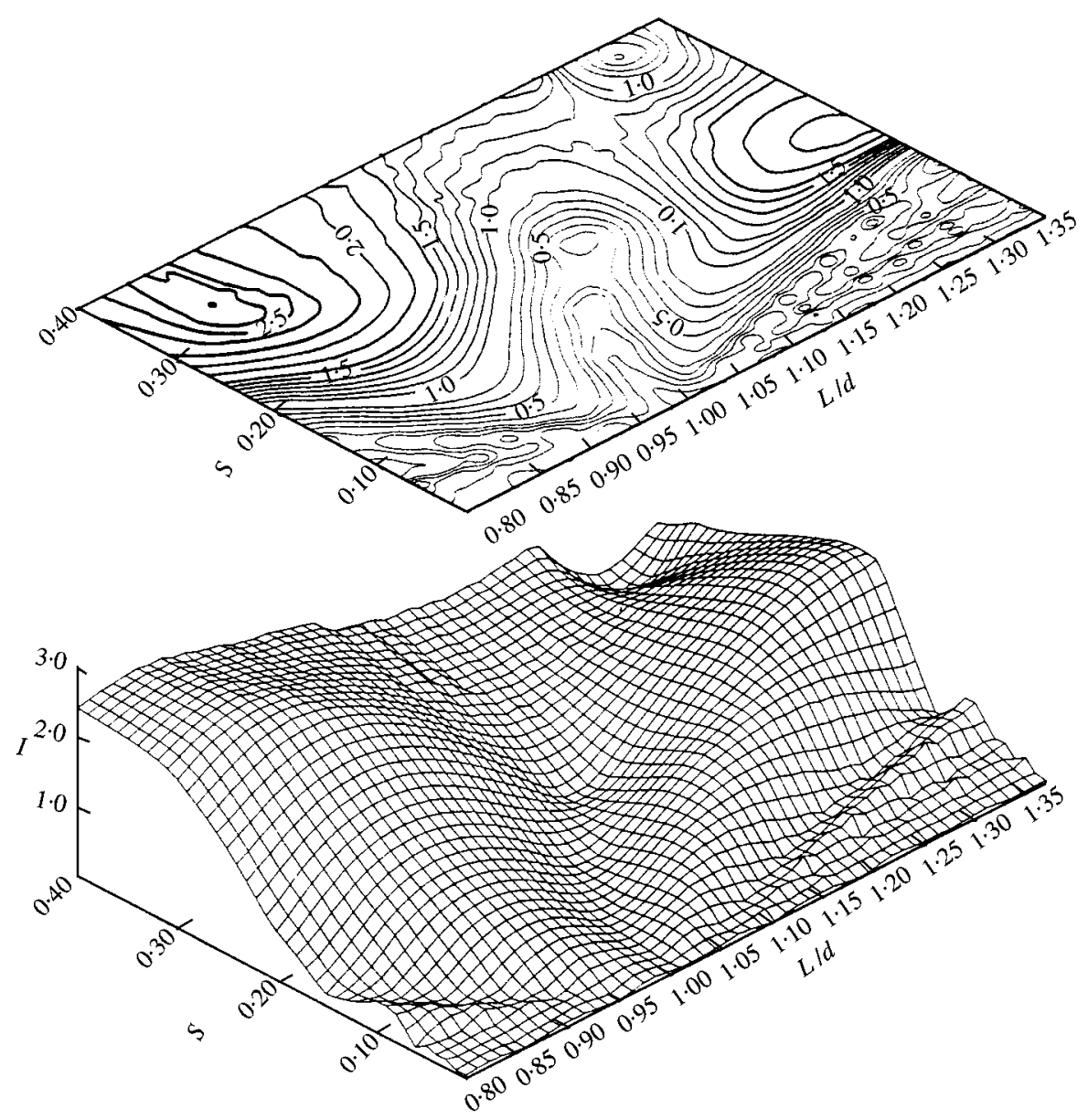

Figure 17. $I-S-L / d$ Diagram for $\xi=0 \cdot 02, \kappa=0 \cdot 25$.

\section{ACKNOWLEDGMENT}

The research reported herein was sponsored in part by the National Science Council through Grant Nos. NSC89-2211-E-002-113 and NSC89-2211-E-032-018.

\section{REFERENCES}

1. S. P. Timoshenko 1922 Philosophical Magazine, Ser. 6, 43, 1018-1019. On the forced vibrations of bridges.

2. J. M. BigGs 1964 Introduction to Structural Dynamics. New York: McGraw-Hill.

3. L. FRỲBA 1972 Vibration of Solids and Structures under Moving Loads. Groningen, The Netherlands: Noordhoff International Publishing.

4. G. B. Warburton 1976 The Dynamic Behaviour of Structures. Oxford: Pergamon.

5. Y. B. YANG, J. D. YAU and L. C. HsU 1997 Engineering Structures 19, 936-944. Vibration of simple beams due to trains moving at high speeds.

6. J. LI and M. SU 1999 Journal of Sound and Vibration, 224, 897-915. The resonant vibration for a simply supported girder bridge under high speed trains. 
7. Y. H. CHEN and C. Y. Li 2000 Journal of Bridge Engineering American Society of Civil Engineers, 5 , 124-130. Dynamic response of elevated high-speed railway.

8. E. S. Hwang and A. S. NowaK 1991 Journal of Structural Engineering American Society of Civil Engineers 117, 1413-1434. Simulation of dynamic load for bridges.

9. M. F. GReEn and D. CEBon 1994 Journal of Sound and Vibration 170, 51-78. Dynamic response of highway bridges to heavy vehicle loads: theory and experimental validation.

10. F. YANG and G. A. FONDER 1996 Earthquake Engineering and Structural Dynamics 25, 195-215. An iterative solution method for dynamic response of bridge-vehicles systems.

11. Y. B. YANG and J. D. YAU 1997 Journal of Structural Engineering American Society of Civil Engineers 123, 1512-1518. Vehicle-bridge interaction element for dynamic analysis.

12. Y. B. YANG, C. H. ChANG and J. D. YAU 1999 International Journal for Numerical Methods in Engineering 46, 1031-1047. An element for analysing vehicle-bridge systems considering vehicle's pitching effect.

13. Y. K. Cheung, F. T. K. Au, D. Y. Zheng and Y. S. Cheng 1999 Journal of Sound and Vibration, 228, 611-628. Vibration of multi-span non-uniform bridges under moving vehicles and trains by using modified beam vibration functions.

14. M. ICHIKAWA, Y. MiYaKaWA and A. MATSUda 2000 Journal of Sound and Vibration 230, 492-506. Vibration analysis of the continuous beam subjected to a moving mass.

15. D. J. Gorman 1975 Free Vibration Analysis of Beams and Shafts. New York: John Wiley \& Sons. 